\title{
Analysis of Hybrid Diversity Combining Technique over TWDP Fading Channel
}

\author{
Ravneet Sodhi and Rajesh Khanna \\ ECE Department, Thapar University, India; \\ ravneet172@yahoo.com,rkhanna2001@gmail.com
}

\begin{abstract}
Background/Objectives: Tremendous growth in the mobile communication services in this technology driven era has made possible for the people to exchange information (data, voice or image) with each other while they are on the move. These wireless systems have brought a revolution in the current market for their high data rates and performance enhanced features. Methods/Statistical Analysis: One of the key issues affecting the performance of a wireless channel is the random variations in the signal strength which may be resulted due to some obstructions like buildings, trees, etc. or due to the movement of the mobile user. This leads to stochastic behavior of the radio channel.. A highly faded Two-Wave with Diffuse Power (TWDP) environment is discussed along with hybrid diversity combining technique. The effectiveness of this scheme is further evaluated by analyzing the system performance in terms of Bit Error Rate (BER) vs Signal to Noise Ratio (SNR). Binary Phase Shift Keying (BPSK) and Quadrature Phase Shift Keying (QPSK) modulation techniques were employed. Findings: It has been shown clearly that TWDP forms the poorer link when compared with other fading channels as BER is higher in this case. Moreover, TWDP fading environment can serve as the worst case scenario in wireless communication system. Also it has been shown as the diversity order increases system's performance is improved in terms of BER and reduction in SNR requirement to achieve a constant BER value. Application/Improvement: This paper presents the need to obtain a clear perception of channel characteristics and to model a system for combating fading and improving the system performance by considering a TWDP fading channel instead of Rayleigh channel while designing a wireless communication system in worst channel conditions..
\end{abstract}

Keywords: Bit Error Rate (BER), Diversity Combining, Maximal Ratio Combining (MRC), Selection Combining (SC), TwoWave with Diffuse Power (TWDP)

\section{Introduction}

Fading and interference are the major bottlenecks of wireless communication scenario which deeply hampers the system performance. Fading refers to the rapid fluctuation or variation in the amplitude, angular phases or multiple delays of the radio signal over a short period of time or short distance, caused due to shadowing from obstacles affecting the radio wave propagation or by the interference of two or more replicas of the transmitted signal that may add destructively or constructively at the receiver end ${ }^{1}$.

Different propagation environments involve different fading characteristics which are entirely diverse and complex. Hence the need of an hour is to design a fading channel model which completely characterizes a particular propagation environment and combat fading as well.

The most commonly used channel models are Rayleigh and Rician models. However, recent research done in ${ }^{2}$ shows that deployed narrow band receivers and direc-

*Author for correspondence 


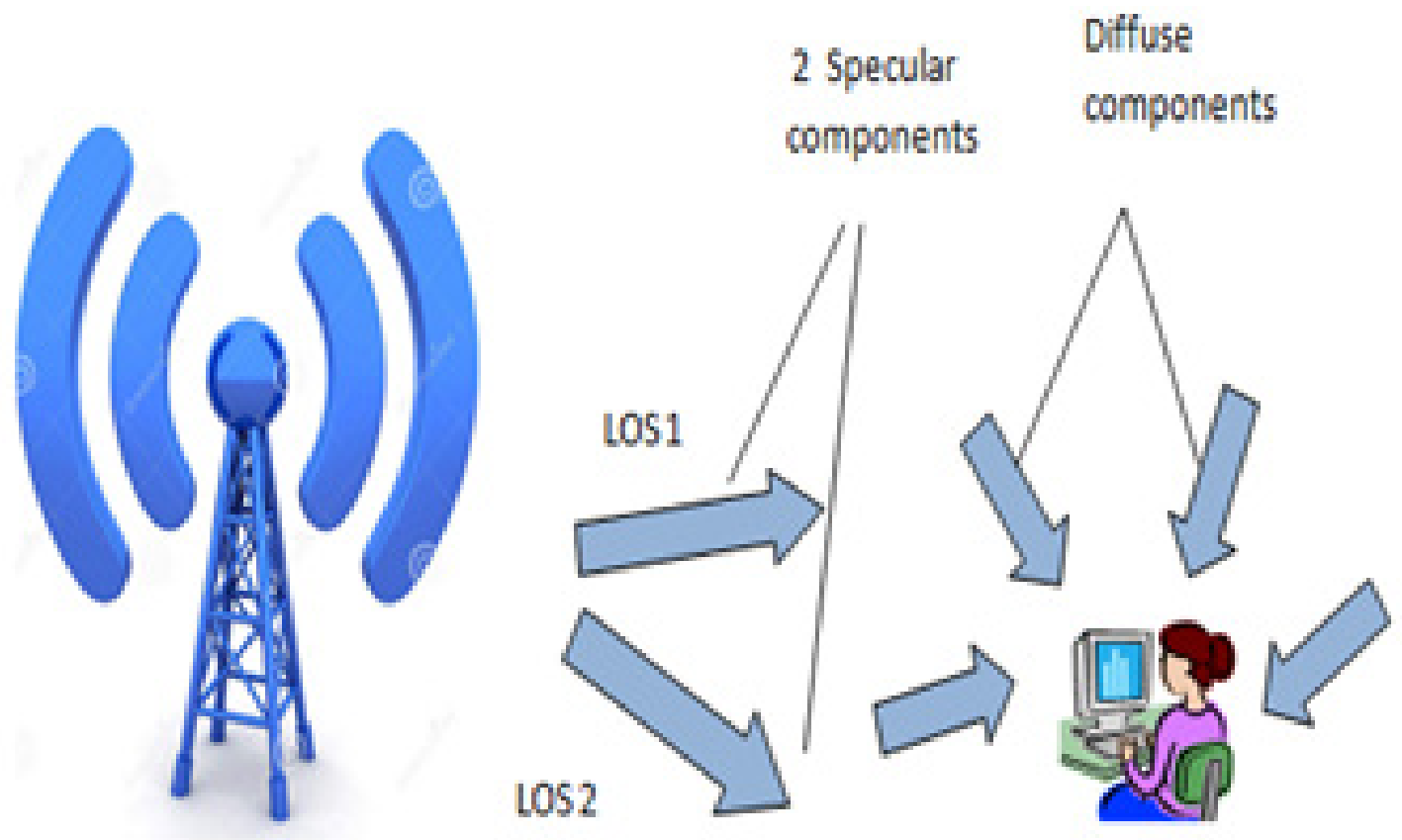

Figure 1. Illustration of two specular components along with many diffuse components in TWDP scenario.

tional antennas form a channel link whose characteristics does not match with those of Rayleigh and Rician fading channels. Novel PDF's describing the small scale fading of two Line-Of-Sight (LOS) components along with diffuse components were developed. A term Order is used in approximating TWDP PDF and thus by increasing this value approximate PDF approaching the exact PDF is shown ${ }^{2}$. Furthermore, it has been depicted in ${ }^{3,4}$ that there exists some Wireless Sensor Network (WSN) applications where sensor nodes are fitted within the cavity environment and in that propagating channel, signal suffer from an even more intense type of fading than Rayleigh channel fading, called as TWDP fading as shown in Figure 1.

Author in [4] further extended the work on hyperRayleigh fading channel by representing it with the most realistic TWDP model. In spite of the fact that Nakagami, log-normal shadowing distribution and Weibull distributions can well characterize the possibility of a severe fading scenario as well as predict infeasible constructive interference. The author proposed that TWDP with $\Delta \approx 1$ and $\mathrm{K}$ lying in the range 0 to $\infty$ is the one and only model to characterize Hyper-Rayleigh fading scenario. TWDP fading occurs when $\mathrm{K}$ parameter i.e. ratio of power of specular component to that of power of diffused one is greater than unity which occurs while using highly directional antennas and wideband signals. These directional antennas help in amplifying the multipath waves in a particular direction while attenuating the others. Wide band signal reception also involves the rejection of multipath components reaching the receiver end at different instants because of different time delays. Authors in ${ }^{5}$ compared the three types of fading namely Rayleigh, Rician and TWDP fading on the basis of different values of $\mathrm{K}$ and $\Delta$. Their work concluded as value of $\mathrm{K}$ tends to infinity and $\Delta$ approaches one, TWDP performance becomes poorer than that of Rayleigh channel. Thus, TWDP can be taken into the consideration so far the worst case scenario is 
concerned while designing a wireless communication system.

An abrupt advancement in the research field of diversity combining in wireless communication has been marked. These strategies assist in employing multipath fading as a boon. These involve transmitting the number of copies of the same information signal through various channels and combining them at the end. This increases the overall Signal to Noise Ratio (SNR) at the receiver side. Pure combining techniques involve single combining technique which is Selection Combining (SC), Maximal Ratio Combining (MRC), Equal Gain Combining (EGC).

In case of MRC, each signal must be aligned in time and equal in phase. These are then weighted optimally with their individual fading amplitudes, and added. It is a complex natured optimal combining scheme because the pre knowledge related to all the channel fading parameters is necessary in this. Moreover, MRC is known to be the most efficient technique as far as performance evaluation is concerned. $\mathrm{In}^{5}$, analysis of Bit Error Rate (BER) of uncoded Binary Phase Shift Keying (BPSK) system in a TWDP fading environment is presented and simulated using MATLAB both with and without Maximal Ratio Combining (MRC) technique. The study further revealed that when MRC receiver is considered, as the SNR value reaches higher and higher coherent combining contributes in improving TWDP's performance above the Rayleigh channel. In $^{6}$, error rate analysis of binary phase shift keying pre-detection MRC systems considering two waves with diffuse power fading channel is given. The authors revealed that the BER of MRC receiver system over a TWDP channel environment is equal to the weighted sum of individual bit error rates of the number of MRC receiver systems in case of Rician fading environment. The results shown provided a basis for the further study of mixed mode fading scenario where different channel links in a diversity system experiences different modes of fading. A new expression of Characteristic Function (CF) of output SNR for TWDP channel condition and PDF expression of SNR for MRC receiver has been obtained in ${ }^{7}$. Analysis of the impact of varying the number of MRC branches on the system performance is also discussed in ${ }^{7}$.
SC is another way of selecting that signal from the branch which has the highest SNR value. The benefit of using SC is that there is no need of coherently summing the individual branch signals. There is a need of considering only the single branch and no additional knowledge regarding the signal phases related to individual branch is needed. The authors in $^{8}$ presented the performance analysis of the simplest form of diversity i.e. Selection Combiner (SC) over the TWDP environment taking into account non-identical and arbitrary fading parameters. They used the PDF expression to derive outage probability expression and BER analysis for both coherent and non coherent modulation techniques and by varying the number of branches and parameters $K$ and $\Delta$. The capacity analysis by deriving the PDF expression of output signal to noise ratio of a selection combiner over the TWDP channel for different adaptive transmission techniques has been provided in ${ }^{9}$. The expressions obtained were in the form of infinite series and contained some special functions like Pochhammer symbol, incomplete gamma function etc. the authors also presented the impact of varying the values of diversity order on the capacity.

Recently Hybrid diversity schemes has gained more importance because of their lesser complexity, lesser consumed power, fewer electronics involved and higher diversity gain offered. A new diversity technique which is a hybrid of both Selection Combining (SC) and Maximal Ratio Combining (MRC) was proposed in ${ }^{10}$. They considered the fading environment to be Rayleigh faded. The system model comprised of $\mathrm{L}$ number of MRC receivers and $\mathrm{M}$ antennas at each receiver. The output of each MRC is sent to SC and a single output is achieved at the end. They derived the PDF of SNR expression for this hybrid technique and hence the outage probability for the same. $\mathrm{In}^{11}$, a new hybrid scheme of diversity MRC/EGC over the Rayleigh faded environment is presented. Performance analysis was done by evaluating outage probability and ABER and PDF of output SNR was found by convolving $L$ fold times the single PDF using MATLAB software.

\section{System Model}

This paper includes a hybrid diversity combining tech- 


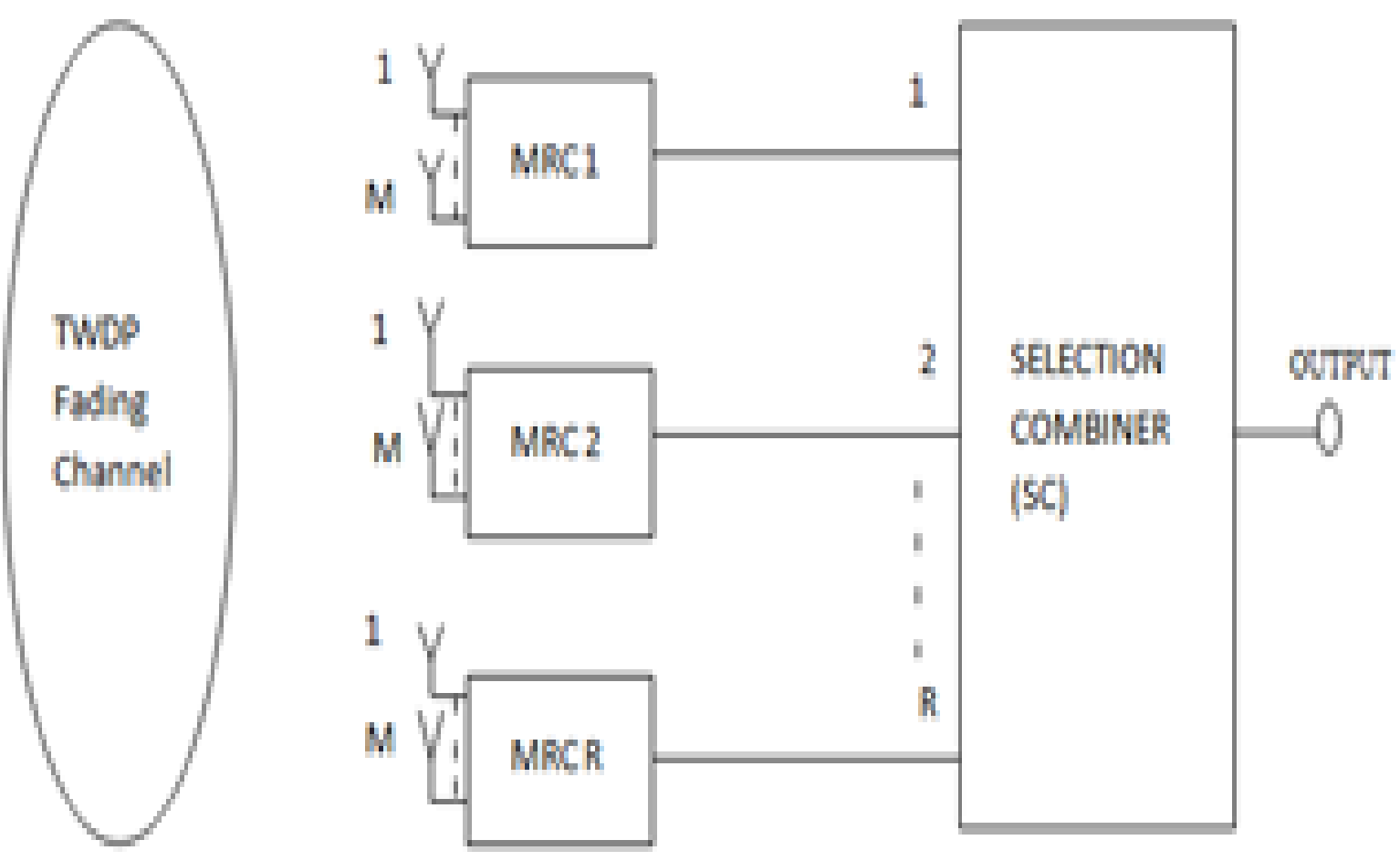

Figure 2. Illustration of hybrid MRC/SC system model in TWDP fading scenario.

nique over the TWDP channel and graphs showing its performance are presented.

In this hybrid system, information signal is received by $\mathrm{R}$ number of MRC receivers each having $\mathrm{M}$ diversity branches. These $\mathrm{M}$ received information signals are weighted and then combined to give maximum value of SNR. From R MRC systems, the one having the highest SNR is chosen using SC with R diversity branches. The system model is presented as shown in Figure 2.

\section{Results and Conclusion}

Simulating the above system model in MATLAB software results in a graph which clearly depicts that as the number of SC combiners are increased from 2 to 4 , the BER value decreases as the SNR is increased i.e. improved performance is seen.

Similarly, this Figure 3 clearly depicts that as the number of MRC receive antennas are increased BER further reduces with increase in SNR. Also at any fixed BER (say $\left.10^{-3}\right)$, SNR requirement is higher for that system having less number of diversity branches.

Thus, from the presented literature review it can be concluded that TWDP fading scenario is the worst case wireless scenario. The hybrid diversity techniques have not been implemented yet with the highly faded TWDP channel. So this model provided an efficient way of utilizing the properties of both MRC and SC combiners to exploit fading as the way to enhance system performance (Figure 4). 


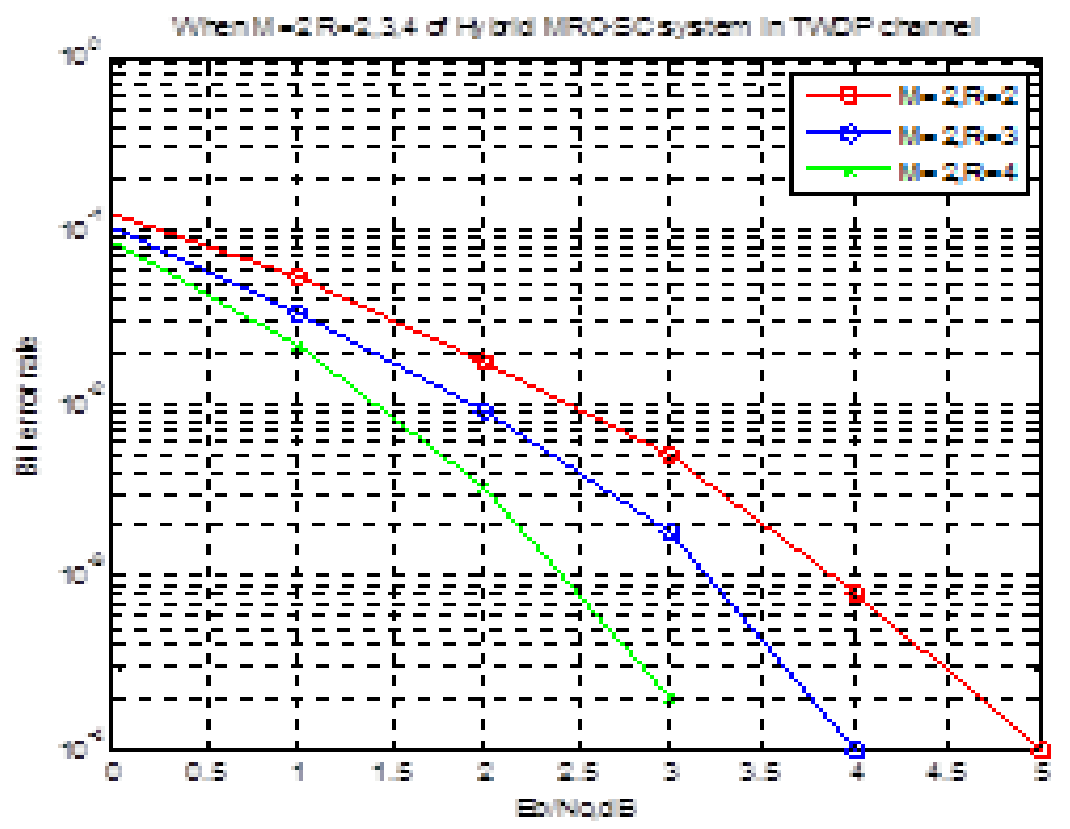

Figure 3. Graph showing the effect of varying $M$ receive antennas and R number of SC in hybrid MRC/SC system.

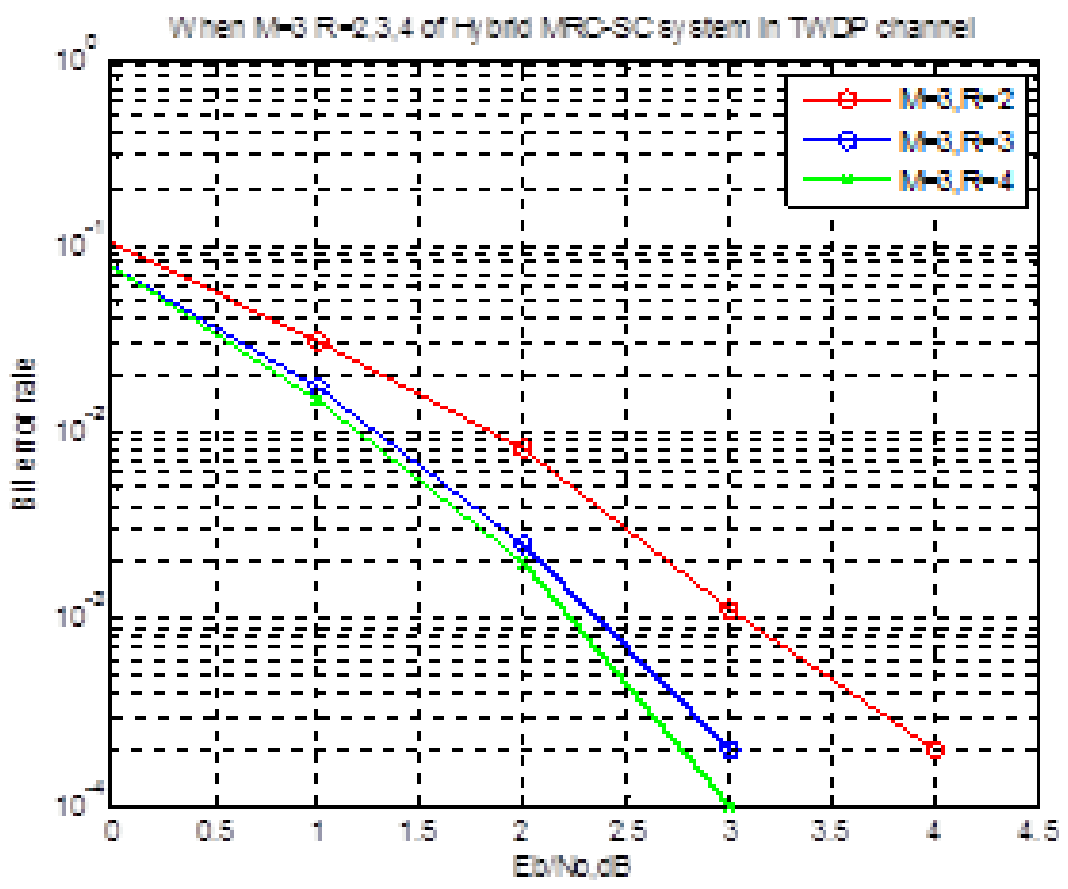

Figure 4. Graph showing the effect of varying $\mathrm{M}$ receive antennas and $\mathrm{R}$ number of SC in hybrid MRC/SC system. 


\section{References}

1. Rappaport T. Wireless Communication, Principles and Practice, 2 ed., Prentice Hall, 2002.

2. Durgin GD, Rappaport TS, de Wolf DA. New analytical models and probability density functions for fading in wireless communication. IEEE Trans. Commun. 2002 Jun; 50(6);1005-15.

3. Frolik J. The case for considering hyper Rayleigh channels. IEEE Trans. Commun. 2007 Apr; 6(4):1235-39.

4. Frolik J. On appropriate Models for characterizing Hyper - Rayleigh Fading., IEEE Transactions on Wireless Communications. 2008 Dec; 7(12):5202-07.

5. Soon H. Oh, Kwok H. Li. BER Performance of BPSK Receivers Over Two Wave with Diffuse Power Fading Channels. IEEE Transactions on Wireless Communications. 2005 Jul; 4( 4):1448-54.

6. Soon H. Oh, Kwok H. Li Performance of BPSK Predetection MRC Systems over Two-Wave with Diffuse Power Fading Channels. IEEE Transactions on Wireless Communications. 2007Aug; 6(8):2772-75.
7. Subadar R, Singh AD. Performance of M MRC receivers over TWDP fading channels. International Journal of Electronics and Communications (AEU). 2014; 68(6):56972 .

8. Subadar R, Singh AD. Performance of SC receivers over TWDP fading channels. IEEE Communication Letters. 2013; 2(3):267-70.

9. Singh AD, Subadar R. Capacity analysis of M-SC receivers over TWDP fading channels. International Journal of Electronics and Communications (AEU). 2014 Feb; 68(2):166-171.

10. Dinamani A, Swagata Das. Performance of a Hybrid MRC/ SC Diversity Receiver over Rayleigh Fading Channel. International Conference on Circuits, Controls and Communications (CCUBE), 2013, p.1-4.

11. Hima Pradeep V, Padmarajan S. Performance Analysis of Rayleigh Fading Channel using the Hybrid TechniqueMRC/EGC. International Journal of Innovative Research in Computer and Communication Engineering. 2015 Oct; 3(10):9136-41. 